\title{
Spatial Uncertainty Propagation Analysis with the spup R Package
}

\author{
by Kasia Sawicka, Gerard B.M. Heuvelink and Dennis J.J. Walvoort
}

\begin{abstract}
Many environmental and geographical models, such as those used in land degradation, agroecological and climate studies, make use of spatially distributed inputs that are known imperfectly. The $R$ package spup provides functions for examining the uncertainty propagation from input data and model parameters onto model outputs via the environmental model. The functions include uncertainty model specification, stochastic simulation and propagation of uncertainty using Monte Carlo (MC) techniques. Uncertain variables are described by probability distributions. Both numerical and categorical data types are handled. The package also accommodates spatial auto-correlation within a variable and cross-correlation between variables. The MC realizations may be used as input to the environmental models written in or called from $\mathrm{R}$. This article provides theoretical background and three worked examples that guide users through the application of spup.
\end{abstract}

\section{Introduction and motivation}

Environmental models that are used to understand and manage natural systems often rely on spatial data as input. Uncertainty in the input data propagates into model predictions. There is a need for systematic analysis of spatial uncertainty propagation in all major inputs and an accounting of spatial auto- and cross-correlation between input uncertainties. Spatial uncertainty propagation analysis has been widely recommended and practised across environmental disciplines, for example, hydrology and water quality (e.g. Hengl et al., 2010; Hamel and Guswa, 2015; Muthusamy et al., 2016; Nijhof et al., 2016; Yu et al., 2016), biogeochemistry (e.g. Boyer et al., 2006; Nol et al., 2010; Pennock et al., 2010; Hugelius, 2012; Vanguelova et al., 2016), or ecology and conservation planning (e.g. Jager et al., 2005; Fisher et al., 2010; Lechner et al., 2014). However, as far as we know, there is no widely applicable software that facilitates such analysis.

Currently, a growing number of software tools are available for uncertainty propagation and uncertainty assessment, some of which have functionality for dealing with spatial uncertainties. These include both free software, like OpenTURNS (Andrianov et al., 2007), Dacota (Adams et al., 2009) and DUE (Brown and Heuvelink, 2007); commercial options, like COSSAN (Schueller and Pradlwarter, 2006); or free packages for licenced software, like the SAFE (Pianosi et al., 2015) or UQLab (Marelli and Sudret, 2014) toolboxes for MATLAB. A broad review of existing software packages are available in Bastin et al. (2013). The use of powerful but complex languages like C++ in Dakota, Python in OpenTURNS or Java in DUE often discourage relevant portions of the scientific community without formal programming skills from the adoption of otherwise powerful tools. There are a number of $\mathrm{R}$ packages relating to uncertainty analysis through sensitivity analysis or use of Bayesian frameworks for model calibration. We have found only three packages: propagate (Spiess, 2014), errors (Ucar, 2017) and metRology (Ellison, 2017) that deal with uncertainty propagation explicitly. However, none of these packages provides functionality for spatial models and variables. Therefore, we undertook a project to develop an $\mathrm{R}$ package that facilitates uncertainty propagation analysis in spatial environmental and geographical modelling.

In this article, we present the spup package for R (Sawicka et al., 2017), which implements a methodology for spatial uncertainty propagation analysis using Monte Carlo (MC) methods. The spup package includes functions for uncertainty model specification, sampling, propagation of uncertainty using MC techniques and examples of results visualization. In this way, it provides support for the entire chain from uncertainty model definition, simulation and propagation to visualization. It is also a generic tool that can handle a great variety of cases. It is suitable for models that consider spatial variables saved in a spatial data frame or raster, as well as non-spatial variables, both continuous and categorical. It works with models written in $\mathrm{R}$ or other programming languages that can be called from $R$. The spup package is intended for researchers and practitioners who recognise the problems of uncertainty in data and models, and are looking for a simple, accessible implementation of a methodology for uncertainty propagation assessment and visualization. At the same time, it is designed to enable more experienced users to easily understand, customise, and possibly further develop the code.

Here, we provide a description of the implemented methodology, describe the package design and illustrate the usage with three simple examples. We demonstrate that the spup package is an effective and easy tool that can be used for multi-disciplinary research, model-based decision support and educational purposes. 


\section{Methodology implemented in spup}

Uncertainty propagation aims to analyse how uncertainty (e.g. from measurement error, sampling, or interpolation) in spatial data, combined with modeling uncertainty (e.g. in model parameters and structure) propagate through the model (Heuvelink et al., 2007). Many environmental and geographical phenomena of interest are spatial in nature and often have strong spatial correlations imposed by the physics and dynamics of the natural systems, making uncertainty evaluation difficult.

A common approach to uncertainty propagation analysis makes use of MC stochastic simulation (Hammersley and Handscomb, 1979; Lewis and Orav, 1989). The MC approach is very flexible and can reach an arbitrary level of accuracy, and is therefore generally preferred over analytical methods such as the Taylor series method (Heuvelink et al., 1989). The MC method for uncertainty propagation analysis consists of the following main steps: (i) uncertainty about the variables is expressed by probability distribution functions (pdfs); (ii) many sets of possible uncertain inputs are generated from their marginal or joint probability distribution using a pseudo-random number generator; (iii) the model is run for each of the simulated input sets; (iv) the set of model outputs forms a random sample from the output pdf, so that the parameters of the output distribution, such as the mean, standard deviation and quantiles, can be estimated from the sample. In other words, the spread in the model outputs characterises how uncertainty about the model inputs has propagated to the model output. Note that the above ignores uncertainty in model parameters and model structure, but these can easily be included if available as pdfs. For example, these might have been obtained through Bayesian calibration (Van Oijen et al., 2005). The above four steps are described in detail further below.

\section{Step 1 - Characterise uncertain model inputs with pdfs}

The most frequently used uncertainty quantification approach represents uncertainty with probability distribution functions (pdfs). In order to represent input uncertainty with a pdf we have to define, for each possible input value, the corresponding probability or probability density. Probability distribution functions can be very complex and can have many parameters. In practice, when information and data from which to derive the pdf are limited, assumptions and simplifications have to be made to obtain reliable estimates of the pdf. In order to reduce the complexity of a pdf for a continuous variable, the distribution function may be described with a simple parametric shape, such as the normal, uniform or lognormal distribution (Bertsekas and Tsitsiklis, 2008). Categorical uncertain variables can be represented by non-parametric distribution, comprising a set of possible outcomes and their probabilities. The probabilities should be non-negative and the sum of the outcome probabilities should equal 1 (Heuvelink, 2007).

Individual variables and the uncertainties associated with them may also be statistically dependent on other variables and their uncertainties. For example, visibility and water vapour in the atmosphere are correlated, and so is the uncertainty between these two properties. Note that correlation between variables as derived from pair observations need not be the same as the correlation between measurement or interpolation errors associated with these variables. For example, elevation and temperature in mountainous area are negatively correlated, while the associated uncertainties may not be correlated at all because of independent measurements methods. Thus, when multiple inputs are uncertain, cross-correlations in their uncertainties must be addressed. In that case, the uncertainty model is described using a joint probability distribution function (jpdf). If the uncertainties are independent, the jpdf is the product of the univariate pdfs and can be estimated by estimating each pdf separately. If the uncertainties are statistically dependent, these dependencies must be estimated alongside the pdfs. In practice, few parametric shapes are available to describe the jpdf of variables that are statistically dependent. For continuous numerical variables, a common assumption is that the multivariate uncertainty model follows a joint-normal distribution with a vector of means $\mu$ and variance-covariance matrix $\Sigma$, where the latter must be square, symmetric and positive-semidefinite.

In different environmental or geographical studies, many variables are spatially distributed and it is likely that there is a relationship between nearby values (i.e. they are auto-correlated). Uncertainty in such cases may be described by the following geostatistical model:

$$
Z(x)=\mu(x)+\sigma(x) \varepsilon(x)
$$

where $\mu(x)$ is the mean of the variable $Z(x)$ at any location $x \in D, D$ is the geographic domain of interest, $\sigma(x)$ is a spatially variable standard deviation associated with $\mu(x)$ (i.e. $\sigma$ parameterises uncertainty such that it may be greater in some regions than in others), and $\varepsilon$ is a zero-mean, unit-variance, second-order stationary residual (i.e. spatial auto-correlation depends only on a distance vector), modelled with a semivariogram or a correlogram (Diggle and Ribeiro, 2007; Webster and Oliver, 2007; Plant, 2012). For defining spatial correlation, spup supports a wide range of positive-definite functions implemented in the gstat package (Pebesma, 2004; Gräler et al., 2016). The mathematical 
background for these calculations is presented in Brown and Heuvelink (2007) and Heuvelink (2007). In case of spatial variables that are cross-correlated with other spatial variables, the linear model of coregionalization (Goovaerts, 1997) is assumed.

Table 1 summarises common assumptions and simplifications in uncertainty propagation analysis for three categories of data: continuous, discrete and categorical. These assumptions are included in the implementation of spup.

\section{Step 2 - Repeatedly sample from spatial pdfs for uncertain inputs}

When an uncertain variable is characterised by a pdf, simulation relies on a pseudo-random number generator. The most common sampling method is independent identically distributed (IID) sampling, in which case each realization is generated as independent draws from the same pdf. In case of a single parameter or univariate distribution, a realization can be easily drawn from various pdfs using algorithms implemented in the stats package in R (see https://cran.r-project.org/web/ views/Distributions. html for details). For a spatially distributed variable (e.g. a DEM) that has a multivariate normal distribution, spup relies on the unconditional Gaussian simulation algorithm implemented in the gstat package (Pebesma, 2004). For simulation from multivariate normal pdfs of non-spatial variables, spup uses the mvtnorm package (Genz et al., 2017; Genz and Bretz, 2009).

In case the input is high-dimensional, straightforward random drawing from the jpdf may not yield a sample that is representative across the whole range of allowable values for each variable, unless the sample size is extremely large. Therefore stratified sampling methods, where values are selected for each variable from each of a pre-specified number of intervals with equal probability mass (Iman and Conover, 1982), are also implemented in spup. The latter can also be done in a spatial context (Pebesma and Heuvelink, 1999).

\section{Step 3 - Run a model with sampled inputs and store model outputs}

Step 3 consists of running a model (e.g. an environmental model) for all sample elements ("simulated realities") generated in Step 2. It is crucial that all models are automated and can be run in batch mode, as in many practical cases, the number of model runs for a Monte Carlo analysis should be at least 100, and often many more (Heuvelink, 1998). In general, the MC sample size must be large enough to guarantee that the outcome of the uncertainty propagation analysis is sufficiently accurate (Heuvelink, 2006). The limitation here is that the required sample size cannot be calculated beforehand because it is derived from the MC output.

It is important to plan what information one needs to retrieve from the set of model outputs. Strategies will vary if the user is interested in the combined or separate effect of uncertain input data or parameters. The simplest approach to estimating the uncertainty contribution of a single uncertain input or parameter is to perform the uncertainty propagation analysis with only the input or, one or more parameters made uncertain. The remaining uncertain parameters are then assumed certain and fixed on their central value. Comparison of the output uncertainties of different inputs (e.g. ratio of variances) provides a measure of the relative contribution. More details on stochastic sensitivity analysis can be found in Saltelli et al. (2000).

Running the model for multiple simulated realities resulting from step 2 may be computationally demanding, particularly when the model is complex and requires much computing time. The computation time can be reduced, as the MC method is very suited for parallel computing with multi-core machines and for grid computing technology (Leyva-Suarez et al., 2015). In this way, computation times can be dramatically reduced. Numerous high-performance and parallel computing packages have been developed in R suiting various approaches. A continuously maintained descriptive list of these is available online at https://cran.r-project.org/web/views/HighPerformanceComputing. html (Eddelbuettel, 2017).

\section{Step 4 - Compute and visualise summary statistics of model outputs}

The resulting set of model outputs forms a random sample from the model output probability distribution. This means that output pdf parameters, such as the mean, median, variance, standard deviation, interquartile range, percentiles, coefficient of variation and threshold exceedance probabilities can be estimated from the sample. For example, the percentiles of the sample can be computed by the quantile() function in R. The accuracy of the sample estimates can also be computed (de Gruijter et al., 2006), although this is currently not implemented in spup. The sample of output values should be stored in case the model output is used as uncertain input for a next model. 


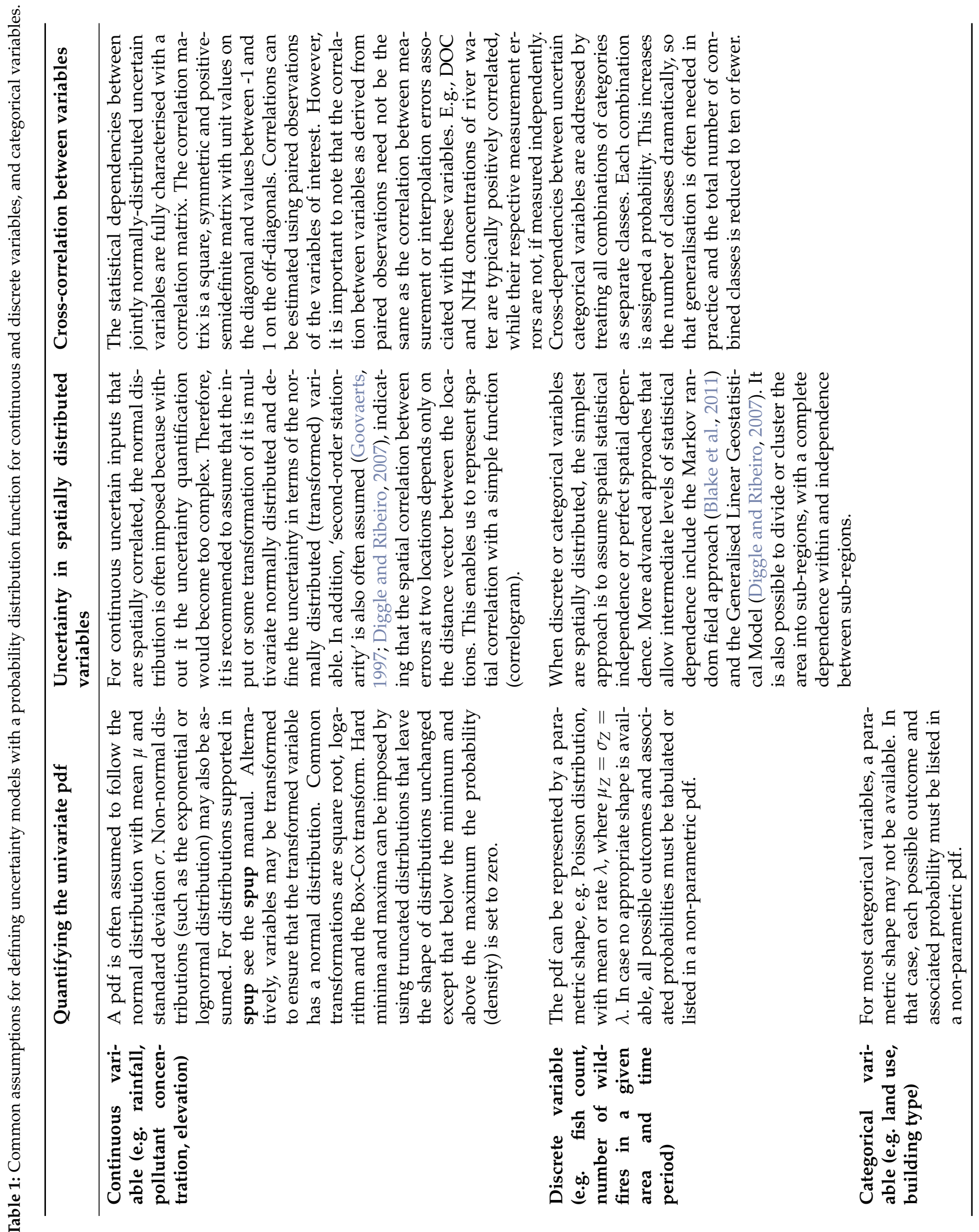


It is important that the uncertainty statistics are communicated in an efficient way that is simultaneously informative and understandable to users that have varied backgrounds in statistics. There should be ample explanation of the results and a visual representation of the uncertainty (MacEachren, 1992; Hengl et al., 2004). For non-spatial and non-temporal data, a number of simple statistical plots can be used to visualise uncertainty represented by probability distributions. If the full probability distribution function is known, this can be visualised in pdf or cumulative distribution function (cdf) plots. Error bars, interquartile range and box plots can be used to show the distribution of values. Uncertainties about categorical data can be shown using stacked bars for the probability for each of the categories, or entropy that provides a summary measure of the spread of probabilities over the categories.

It is important to distinguish between presentation techniques and presentation modes for uncertaintly visualisation of spatial data. General presentation techniques are (i) adjacent maps, (ii) sequential presentation and (iii) bi-variate maps (MacEachren et al., 2005). These techniques can be presented in three modes: static, dynamic and interactive (Kinkeldey et al., 2014). A common example of adjacent maps for continuous variables is to put maps of the mean and standard deviation next to each other. For visualising categorical attribute uncertainty, the simplest method is also using adjacent maps, for example by showing a map of the the most probable category next to a map of the probability of that category. Sequential presentation may be shown in dynamic mode.

\section{The spup package}

The spup package is developed in $\mathrm{R}$ to provide a simple solution for those who wish to include uncertainty analysis in their studies, with emphasises on studies including spatial variables. The spup package implements the methodology described in Section Methodology implemented in spup. We describe spup version 1.3-1 available on CRAN. The development version is available on gitHub at https://github.com/ksawicka/spup. The package is freely available under the GPL3.

Three example datasets are provided with the spup package:

1. Digital Elevation Model of Zlatibor region in Serbia $(3 \times 4.5 \mathrm{~km}, 30 \mathrm{~m}$ resolution) (Hengl et al., 2008)

2. Soil organic carbon and total nitrogen content of the south region of Lake Alaotra in Madagascar (33x33km, 250m resolution) (Hengl et al., 2017)

3. Rotterdam neighbourhood buildings distribution (Kadaster, The Netherlands).

These datasets are used in Section Application examples and in the vignettes included in the package. Additionally, the package includes simple $\mathrm{R}$ and $\mathrm{C}$ functions that represent environmental models used in examples.

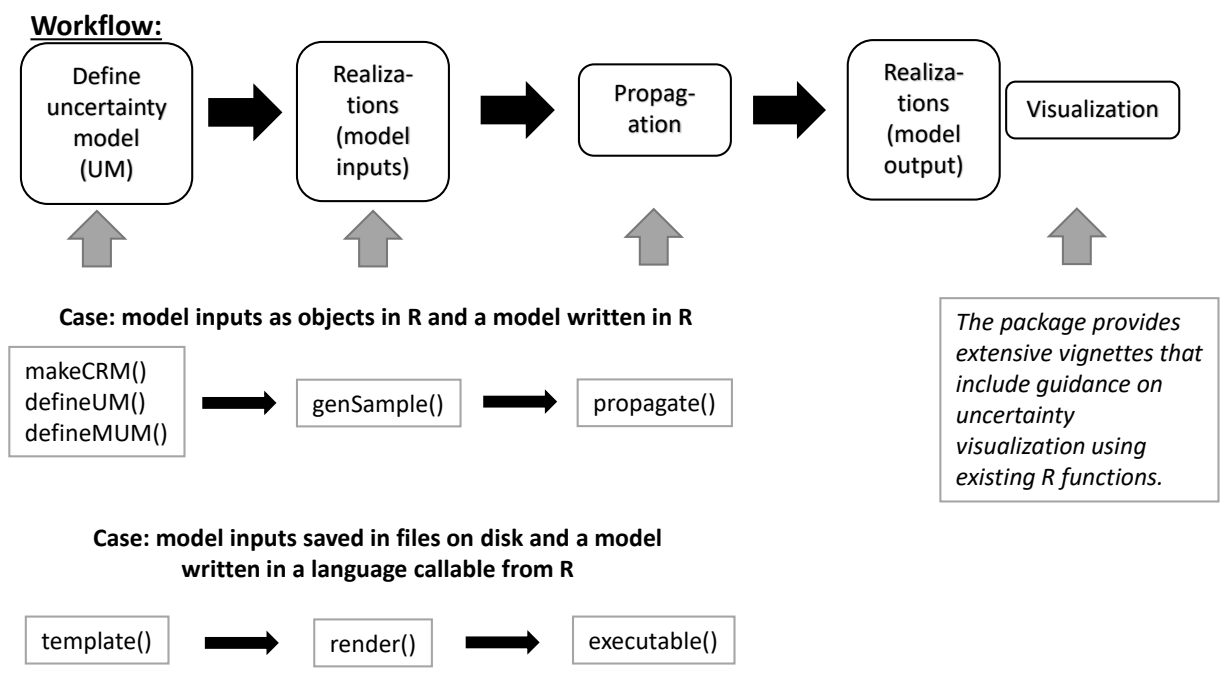

Figure 1: Flowchart presenting workflow with functions implemented in spup.

Figure 1 presents a flowchart with the workflow and key functions implemented in spup. The package is designed in a way that the key functions correspond with the steps presented in Section Methodology implemented in spup. The output of functions for defining the uncertainty model 
becomes an input to functions for generating a MC sample. The generated MC sample in turn is used as input to functions facilitating propagation of uncertainty. Qualitative descriptions of the key functions are given in Table 2. More details about functions arguments and output can be found in the package manual (Sawicka et al., 2017) and in the examples below.

Table 2: Key functions in package spup.

\begin{tabular}{|c|c|}
\hline Function & Description \\
\hline makeCRM() & Define a spatial correlogram model. \\
\hline defineUM() & $\begin{array}{l}\text { Define marginal uncertainty distributions for model input- } \\
\text { s/parameters for subsequent Monte Carlo analysis. Output } \\
\text { class depends on the arguments provided. }\end{array}$ \\
\hline defineMUM() & $\begin{array}{l}\text { Allows the user to define joint uncertainty distributions for } \\
\text { continuous model inputs/parameters for subsequent Monte } \\
\text { Carlo analysis. }\end{array}$ \\
\hline genSample() & $\begin{array}{l}\text { Methods for generating a Monte Carlo sample of uncertain } \\
\text { variables. }\end{array}$ \\
\hline template() & $\begin{array}{l}\text { Defines a "container" class to store all templates with model } \\
\text { inputs. }\end{array}$ \\
\hline executable() & $\begin{array}{l}\text { Produces an } \mathrm{R} \text { function wrapper around a model that can be } \\
\text { called using system } 2() \text { from } \mathrm{R} \text {. }\end{array}$ \\
\hline render () & $\begin{array}{l}\text { Replaces the tags in moustaches in template file or character } \\
\text { object by text. }\end{array}$ \\
\hline propagate() & $\begin{array}{l}\text { Runs the model repeatedly with Monte Carlo realizations of } \\
\text { the uncertain input/parameters. }\end{array}$ \\
\hline
\end{tabular}

\section{Application examples}

\section{Example 1 - Uncertainty propagation analysis with auto- and cross- correlated variables}

In many geographical studies, variables are cross-correlated. As a result, uncertainty in one variable may be statistically dependent on uncertainty in the other. For example, soil properties such as organic carbon (OC) and total nitrogen (TN) content are cross-correlated. These variables are used to derive the $\mathrm{C} / \mathrm{N}$ ratio, which is important information to evaluate soil management and increase crop productivity. Errors in OC and TN maps will propagate into the $\mathrm{C} / \mathrm{N}$ ratio map. The cross-correlation between the uncertainties in $\mathrm{OC}$ and $\mathrm{TN}$ will affect the degree of uncertainty in the $\mathrm{C} / \mathrm{N}$ ratio.

The example data for $\mathrm{C} / \mathrm{N}$ calculations are a $250 \mathrm{~m}$ resolution mean $\mathrm{OC}$ and $\mathrm{TN}$ of a $33 \mathrm{~km} \times 33 \mathrm{~km}$ area adjacent to lake Alaotra in Madagascar (Figure 2) (Hengl et al., 2017). The datasets include four spatial objects: a mean OC and TN of the area, with their standard deviations assumed to be equal to $10 \%$ of the mean.

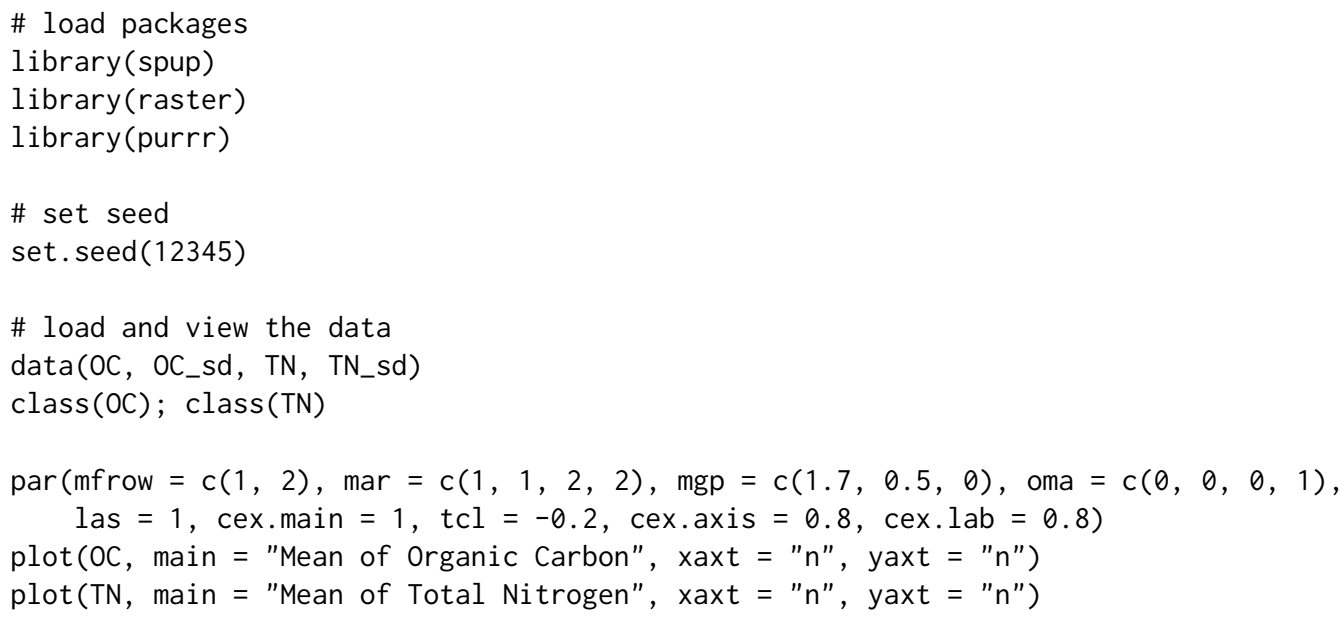

The first step in uncertainty propagation analysis is to define an uncertainty model for the uncertain input variables, here OC and TN. First, the marginal uncertainty model is defined for each variable separately, and next the joint uncertainty model is defined for the variables together. In case of OC and TN, the $\varepsilon$ (Eq. 1) are spatially correlated. For each of the variables, the makeCRM() function collates all necessary information into a list. We assume that the spatial autocorrelation of the OC and TN 
Mean of Organic Carbon

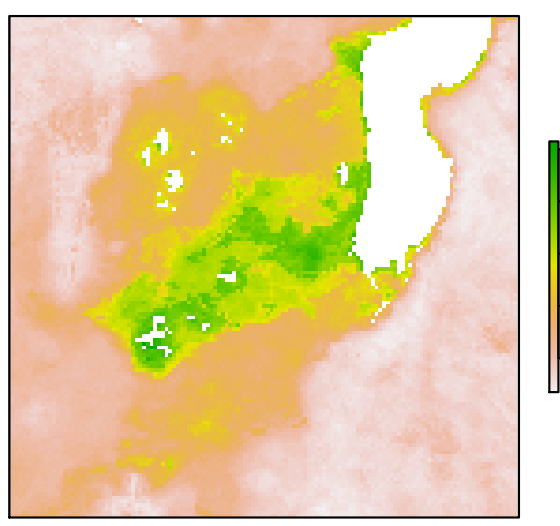

Mean of Total Nitrogen

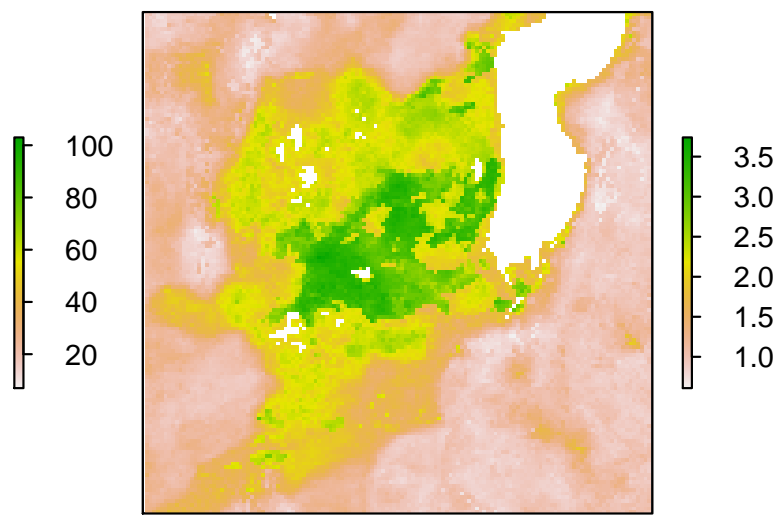

Figure 2: Maps of means of soil organic carbon and soil total nitrogen near lake Alaotra in Madagascar.

errors are both described by a spherical correlation function with a short-distance correlation of 0.6 for OC and 0.4 for TN, with a range parameter of $1000 \mathrm{~m}$ (Figure 3). It is important at this step to ensure that the correlation function types as well as the ranges are the same for each variable. This is a requirement for further analysis, because spup employs the linear model of co-regionalization (Wackernagel, 2003).

\# define spatial correlogram models

OC_crm $<-$ makeCRM $($ acfo $=0.6$, range $=1000$, model $=$ "Sph" $)$

TN_crm $<-$ makeCRM $(\operatorname{acf0}=0.4$, range $=1000$, model $=$ "Sph" $)$

$\operatorname{par}(\operatorname{mfrow}=c(1,2), \operatorname{mar}=c(3,2.5,2,1), \operatorname{mgp}=c(1.7,0.5,0)$, oma $=c(0,0,0,0)$,

las $=1$, cex.main $=1$, tcl $=-0.2$, cex.axis $=0.8$, cex.lab $=0.8$ )

plot (OC_crm, main = "OC correlogram")

$\operatorname{plot}\left(T_{N} \_c r m\right.$, main $=$ "TN correlogram")
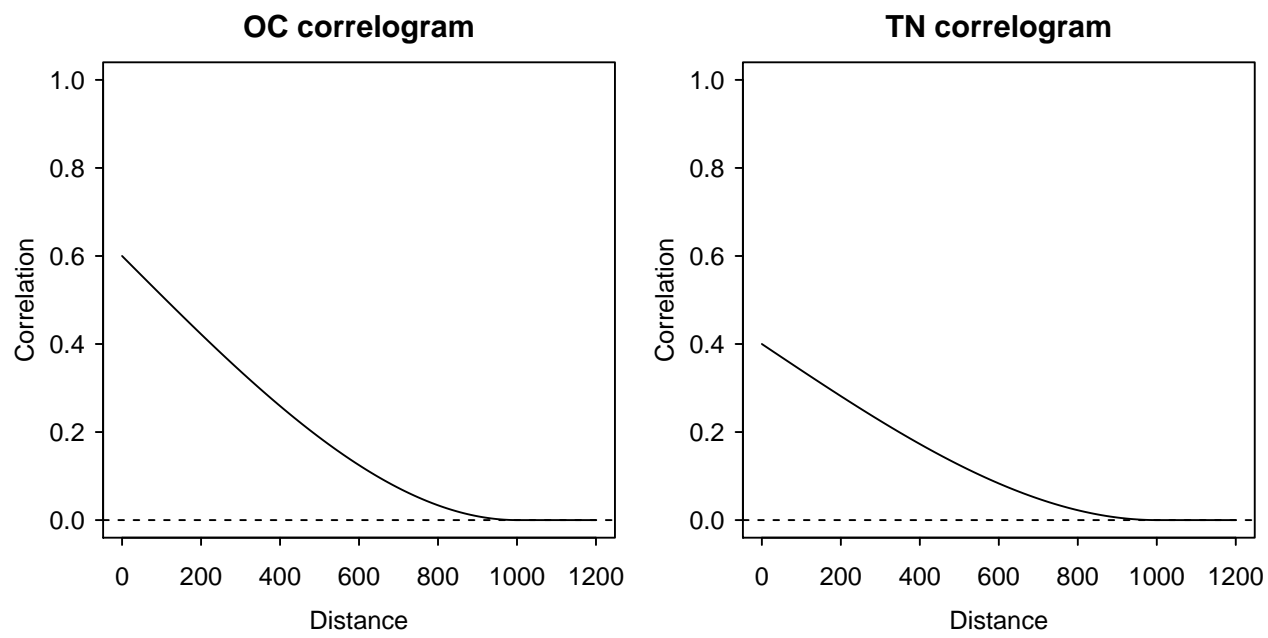

Figure 3: Spatial correlograms of soil OC and TN in the study area.

Spatial correlograms summarise patterns of spatial autocorrelation in data and model residuals. They show the degree of correlation between values at two locations as a function of the separation distance between the locations. In the case above, as is usually the case, the correlation declines with distance. Here, the correlation is zero for distances greater than $1000 \mathrm{~m}$. More information about correlograms is included in the spup DEM vignette. In order to complete the description of each individual uncertain variable, the defineUM() function collates all information about the OC and TN uncertainty.

\# define uncertainty model for the $\mathrm{OC}$ and TN 


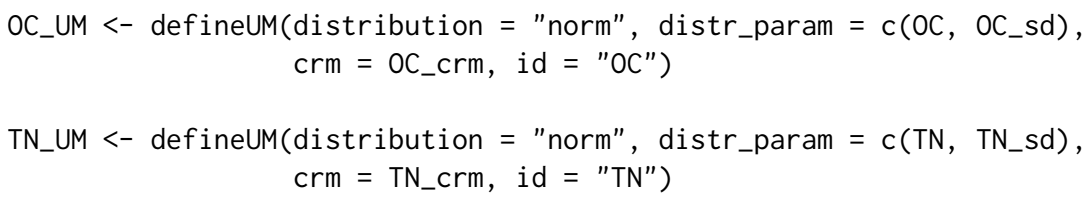

class (OC_UM)

[1] "MarginalNumericSpatial"

class(TN_UM)

[1] "MarginalNumericSpatial"

Both variables are of the same class "MarginalNumericSpatial". This is one of the requirements for defining a multivariate uncertainty model. The defineMUM() function collates information about uncertainty in each variable, and information about their cross-correlation.

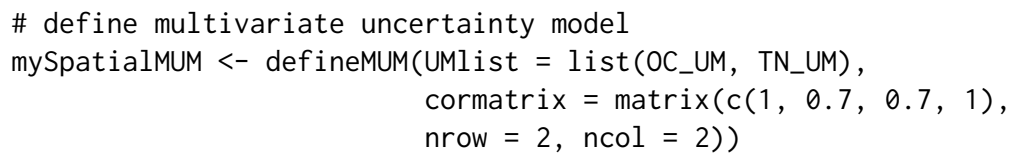

class (mySpatialMUM)

[1] "JointNumericSpatial"

In this example, a geostatistical model for $\mathrm{OC}$ and $\mathrm{TN}$ is defined that assumes multivariate normality and has spatial correlation functions as follows:

$$
\begin{aligned}
& \rho_{\text {OC }}(h)=1 \text { for } h=0 \\
& \rho_{O C}(h)=0.6 \cdot\left\{1-\frac{3}{2} \frac{h}{1000}+\frac{1}{2}\left(\frac{h}{1000}\right)^{3}\right\} \text { for } 0<h<1000 \\
& \rho_{O C}(h)=0 \text { for } h \geq 1000 \\
& \rho_{T N}(h)=1 \text { for } h=0 \\
& \rho_{T N}(h)=0.4 \cdot\left\{1-\frac{3}{2} \frac{h}{1000}+\frac{1}{2}\left(\frac{h}{1000}\right)^{3}\right\} \text { for } 0<h<1000 \\
& \rho_{T N}(h)=0 \text { for } h \geq 1000
\end{aligned}
$$

where $h$ is Euclidean distance and where the mathematical definition of the spherical correlation function (i.e., the semivariogram) has been used (Webster and Oliver, 2007).

Generating possible realities of the selected variables is completed by using the genSample() function:

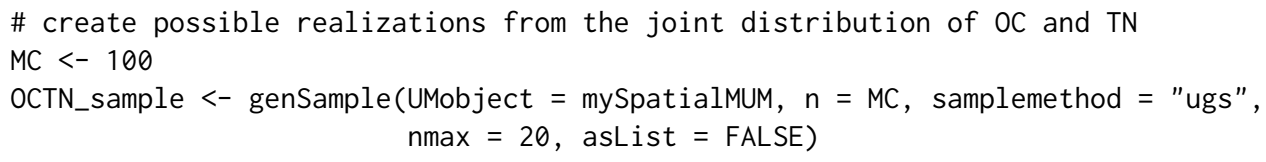

Note the argument asList has been set to FALSE. This indicates that the sampling function will return an object of a class of the distribution parameters class. This is useful if there is a need to visualise the sample or compute summary statistics quickly.

A first assessment of uncertainty is done by plotting the means and standard deviations of the sampled OC and TN. Note that if the sample size is very large, then the sample mean and standard deviation would approximate the mean and standard deviation (Figure 4).

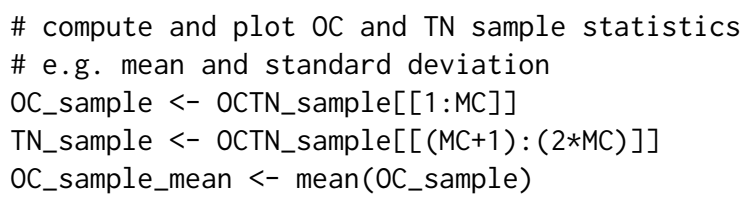


TN_sample_mean <- mean (TN_sample)

OC_sample_sd <- calc(OC_sample, fun = sd)

TN_sample_sd $<-$ calc $\left(T N \_s a m p l e\right.$, fun $=$ sd $)$

$\operatorname{par}(\operatorname{mfrow}=c(1,2), \operatorname{mar}=c(1,1,2,2), \operatorname{mgp}=c(1.7,0.5,0)$, oma $=c(0,0,0,1)$,

las $=1$, cex. main $=1$, tcl $=-0.2$, cex. axis $=0.8$, cex.lab $=0.8$ )

plot (OC_sample_mean, main $=$ "Mean of OC realizations", xaxt $=" n$ ", yaxt $=$ " $n "$ )

plot (TN_sample_mean, main $=$ "Mean of TN realizations", $x a x t=" n "$, yaxt $=" n "$ )

Mean of $\mathrm{OC}$ realizations

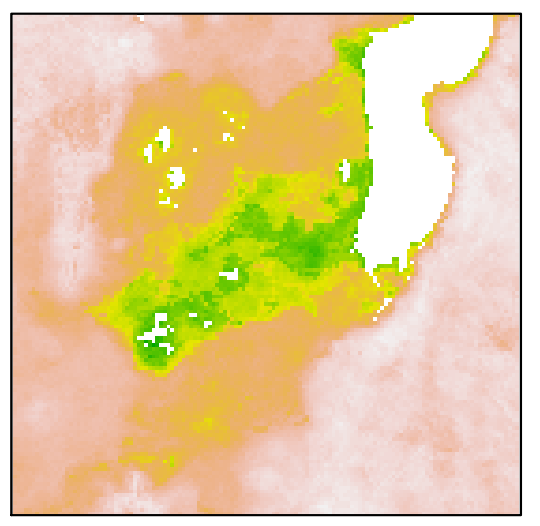

Mean of TN realizations

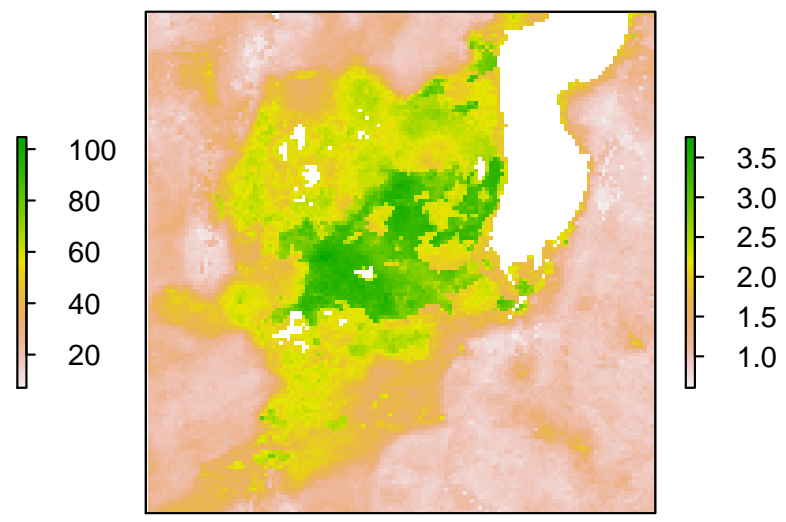

Figure 4: Maps of means of sampled realizations of OC and TN.

In order to perform uncertainty propagation analysis using spup, the model through which uncertainty is propagated needs to be defined as an $\mathrm{R}$ function:

\# $\mathrm{C} / \mathrm{N}$ model

C_N_model_raster <- function (OC, TN) OC/TN

The propagation of uncertainty occurs when the model is run with uncertain inputs. Running the model with a sample of realizations of uncertain input variable(s) yields an equally large sample of model outputs that can be further analysed. We use the propagate() function to run the $\mathrm{C} / \mathrm{N}$ ratio model with the OC and TN realizations. To run propagate(), the samples of the uncertain input variables must be saved in lists and then collated into a list of lists. The existing OCTN_sample object can be coerced or generated automatically by setting asList = TRUE in genSample().

\# coerce a raster stack to a list

$1<-\operatorname{list}()$

$1[[1]]<-\operatorname{map}\left(1: 100\right.$, function $(x)\left\{0 C T N \_\right.$sample$\left.\left.[[x]]\right\}\right)$

$1[[2]]<-\operatorname{map}\left(101: 200\right.$, function $\left.(x)\left\{0 C T N \_s a m p l e[[x]]\right\}\right)$

OCTN_sample $<-1$

\# run uncertainty propagation

CN_sample $<-$ propagate (realizations $=$ OCTN_sample, model $=$ C_N_model_raster, $\mathrm{n}=\mathrm{MC}$ )

Uncertainty in $\mathrm{C} / \mathrm{N}$ predictions can be visualised by calculating and plotting the relative error (Figure 5). The relative error gives an indication of the accuracy relative to its size.

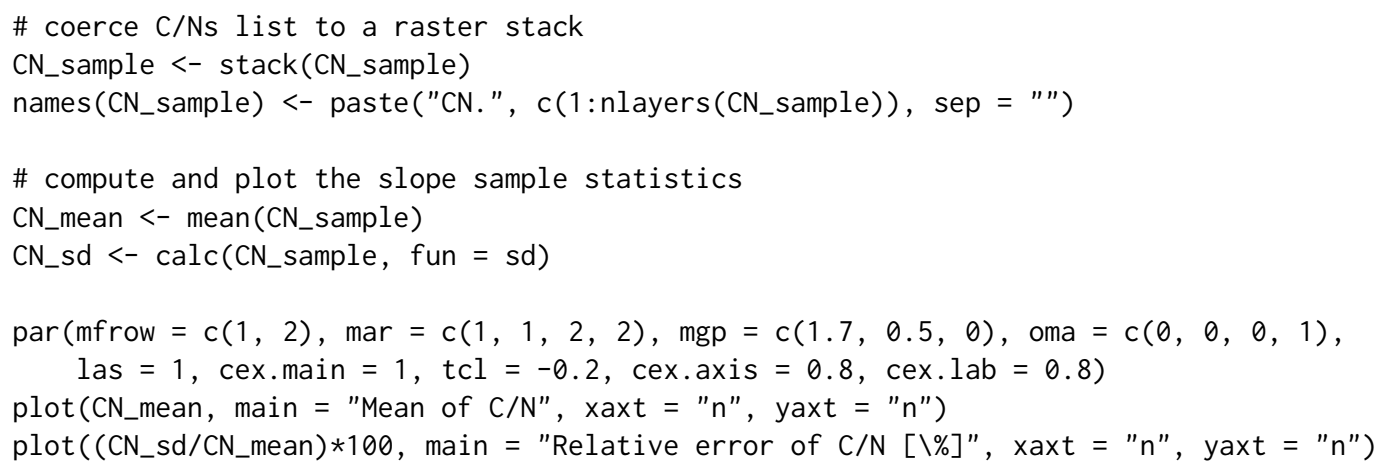


Mean of $\mathbf{C} / \mathbf{N}$

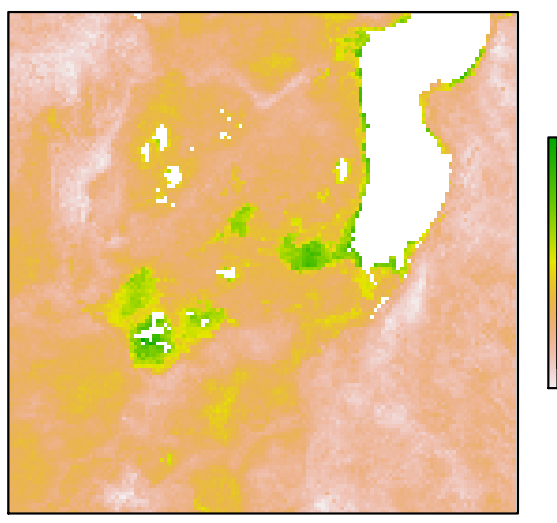

Relative error of $\mathrm{C} / \mathrm{N}[\%]$

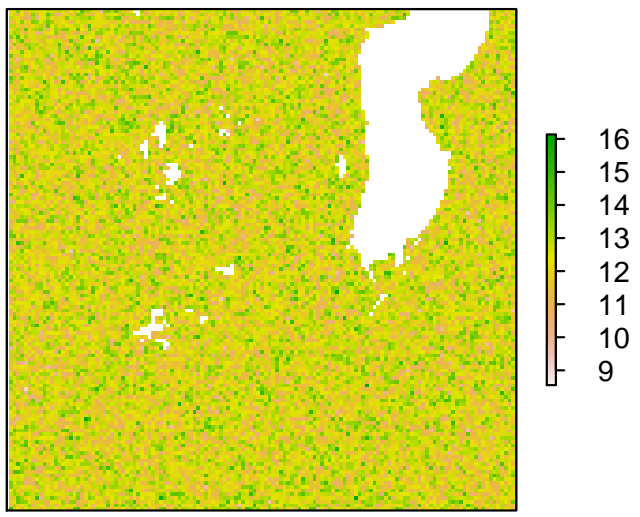

Figure 5: Maps of standard deviation and relative error of $\mathrm{C} / \mathrm{N}$ ratio sample in the study area.

Further analysis may include identification of all locations where the $\mathrm{C} / \mathrm{N}$ ratio is in a specific range with decreasing probability. For example identifying areas where the $\mathrm{C} / \mathrm{N}$ ratio is higher than 20 might help farmers to identify which plots require action to improve soil quality (Figure 6).

\# calculate quantiles

CN_sample_df <- as(CN_sample, "SpatialGridDataFrame")

CN_q <- quantile_MC_sgdf(CN_sample_df, probs $=c(0.01,0.1,0.5,0.9)$, na.rm $=$ TRUE $)$

CN_q\$good4crops99perc <- ifelse $\left(C N \_q \$ p r o b 1\right.$ perc $\left.>20,1,0\right)$

CN_q\$good4crops90perc <- ifelse $\left(C N \_q \$\right.$ prob10perc $\left.>20,1,0\right)$

CN_q\$good4crops50perc <- ifelse $\left(C C N_{-}\right.$q\$prob50perc $\left.>20,1,0\right)$

CN_q\$good4crops10perc <- ifelse $\left(C C_{-} q \$\right.$ prob90perc $\left.>20,1,0\right)$

CN_q\$good4crops <- CN_q\$good4crops99perc + CN_q\$good4crops90perc +

CN_q\$good4crops50perc + CN_q\$good4crops10perc

CN_q\$good4crops[CN_q\$good4crops == 4]<- "No improvement needed"

CN_q\$good4crops[CN_q\$good4crops == 3] <- "Possibly improvement needed"

CN_q\$good4crops[CN_q\$good4crops == 2] <- "Likely improvement needed"

CN_q\$good4crops[CN_q\$good4crops == 1]<- "Definitely improvement needed"

CN_q\$good4crops[CN_q\$good4crops == 0] <- "Definitely improvement needed"

CN_q\$good4crops <- factor $\left(C N \_q \$ g o o d 4 c r o p s\right.$,

levels = c("Definitely improvement needed",

"Likely improvement needed",

"Possibly improvement needed",

"No improvement needed"))

spplot(CN_q, "good4crops", col.regions = c("red3", "sandybrown", "greenyellow",

"forestgreen"), main = "Areas with sufficient $\mathrm{C} / \mathrm{N}$ for cropping")

\section{Example 2 - Uncertainty propagation analysis with categorical data}

In many aspects of life, information take the form of categorical data. For example, in a city neighbourhood or a district, each building may be assigned a function such as housing, office, recreation, or other. The city council may impose different tax levels depending on the building function. Suppose that the function of a building is assigned depending on the percentage of the building used for living (i.e., the residential use) and number of addresses in the building. If the residential area is greater than $80 \%$ and has at least one address registered, then the building is classified as "residential". If the residential area is smaller than $80 \%$ and at least one address is present, then the building is classified as "office". If no address is present, the building function is classified as "other". The city council imposes annual tax of $€ 1000$ for residential buidings, $€ 10000$ for office buildlings, and $€ 10$ for other buildings. The $80 \%$ threshold is approximate, and some buildings classified as "other" could have an assigned 


\section{Areas with sufficient $\mathrm{C} / \mathrm{N}$ for cropping}

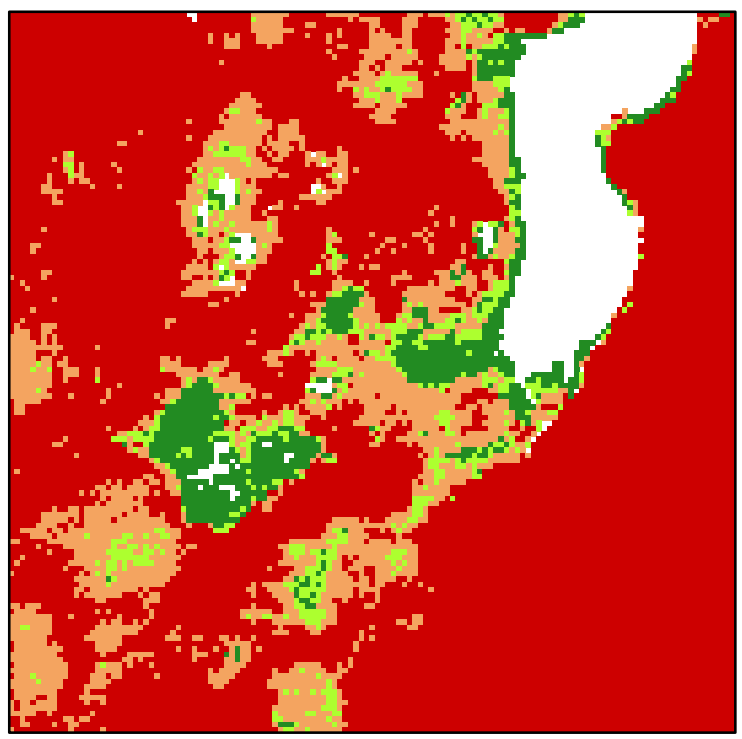

No improvement needed

Possibly improvement needed

Likely improvement needed

Definitely improvement needed

Figure 6: Map of soil quality classification (depending on $\mathrm{C} / \mathrm{N}$ ratio) for crop production.

address that has not yet been entered into the tax system. Therefore, the council wishes to calculate the uncertainty in tax revenue introduced by erroneous building classification. For this example, we will use the spatial distribution of buildings in a neighborhood of Rotterdam, NL (Figure 7).

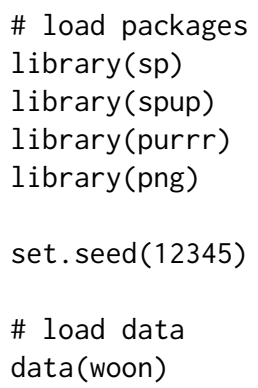


colorkey $=$ FALSE, key. space $=\operatorname{list}(x=0.1, y=0.93$, corner $=c(0,1))$, sp.layout $=$ list (scale, text1, text2, arrow, NL_map), main = "Neighbourhood in Rotterdam, $\mathrm{NL} "$ )

\section{Neighbourhood in Rotterdam, NL}

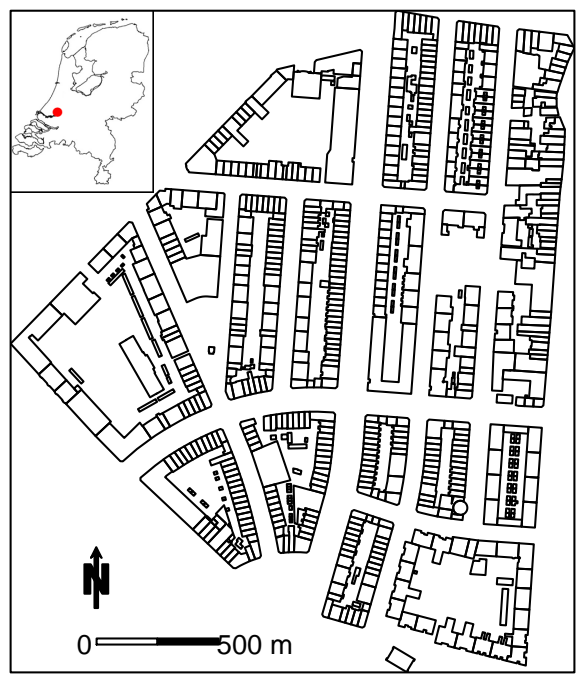

Figure 7: Map of a neighbourhood of Rotterdam used as study area. Contours represent buildings.

The woon object is a SpatialPolygonDataFrame where each building is represented by one polygon (Figure 7). The attributes include:

- vbos: The number of addresses present in the building

- woonareash: The percentage residential area

- Function: The assigned category depending on vbos and woonareash, where 1 represents residental; 2, office, and 3, other.

- residential: Probability that the building is residential

- office: Probability that the building is an office buildling

- other: Probability that the building has another function

Figure 8 illustrates the probabilities associated with each building for the three possible categories.

\# plot probabilities for each polygon

spplot (woon $[c(4,5,6)]$ )

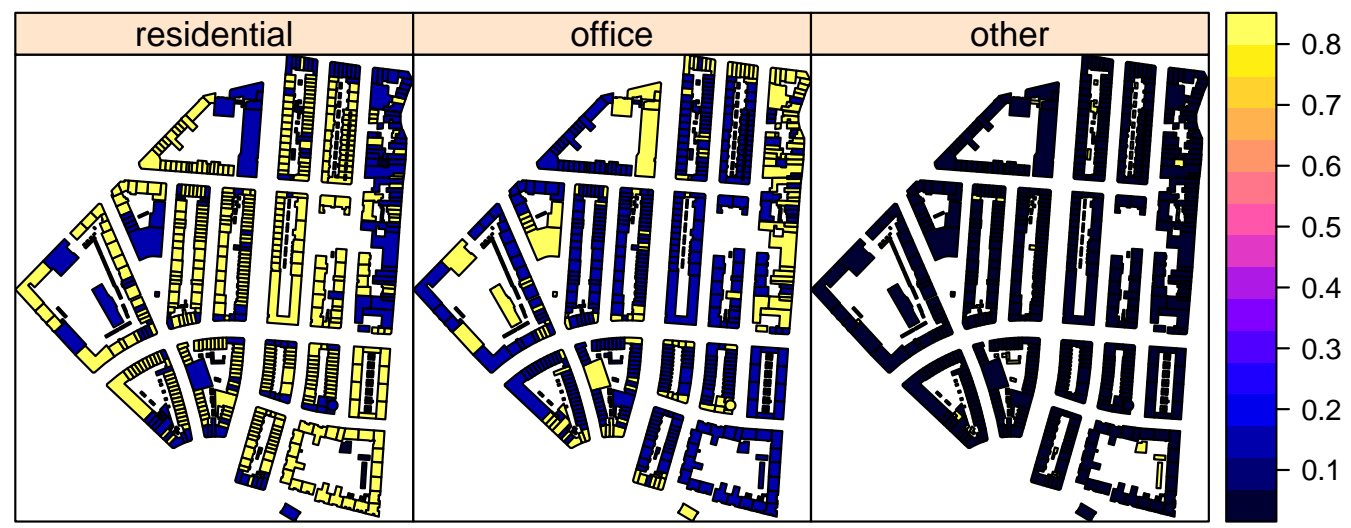

Figure 8: Probabilities of correct classification of each building. 
In case of categorical variables, the uncertainty is described by a non-parametric pdf. In this case, it is a vector of probabilities that a building belongs to a certain category. In case of spatially distributed variables, this must be done for each polygon, hence the dataset has maps of these probabilities saved in the same object. We further assume that uncertainties are spatially independent, implying that the joint pdf of all buildings in the neighbourhood is the product of the marginal pdfs for all buildings. Thus, we can generate possible realities for all buildings independently. To unite all information of the uncertainty model for the building function, we use the defineUM() function that collates all information into one object.

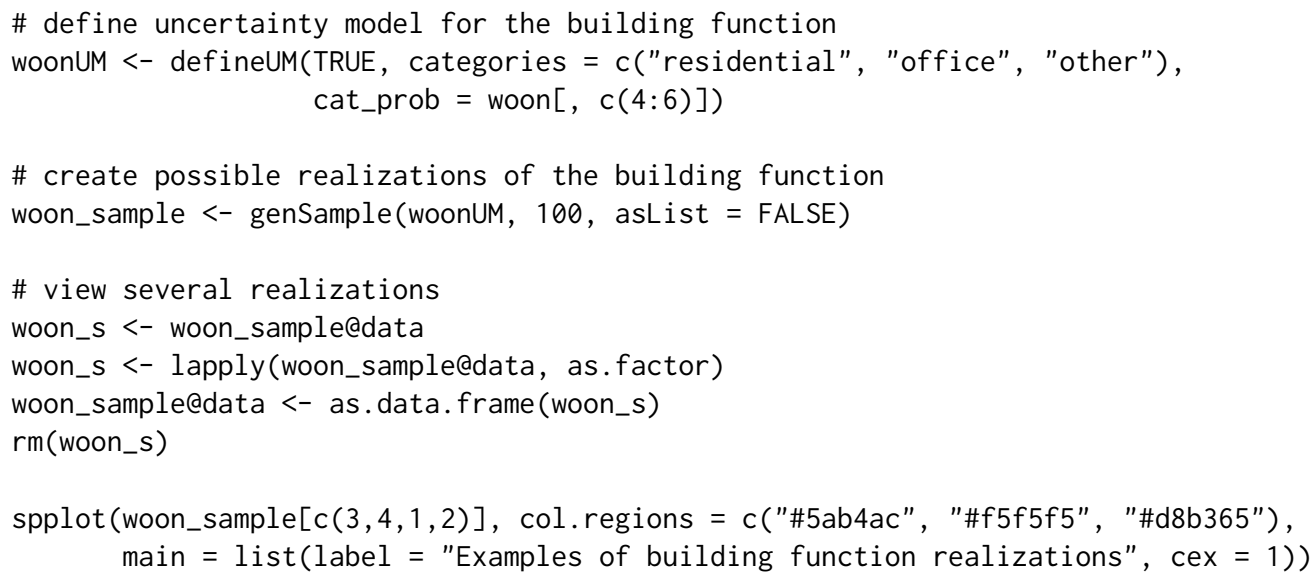

\section{Examples of building function realizations}

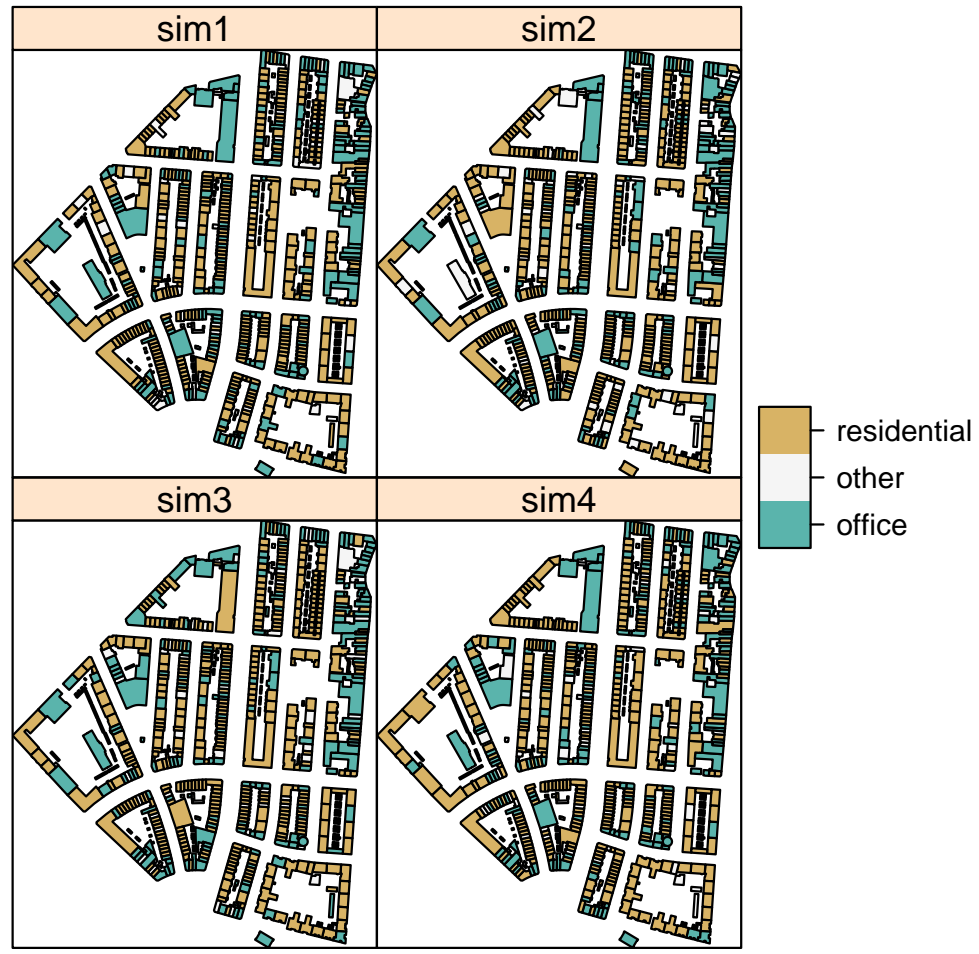

Figure 9: Examples of Monte Carlo realizations of possible classifications for each building.

Examples of building functions Monte Carlo realizations are shown in Figure 9.

To propagate uncertainty, we run the model repeatedly with the sample created above. The tax model is defined as:

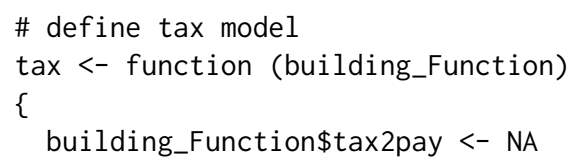




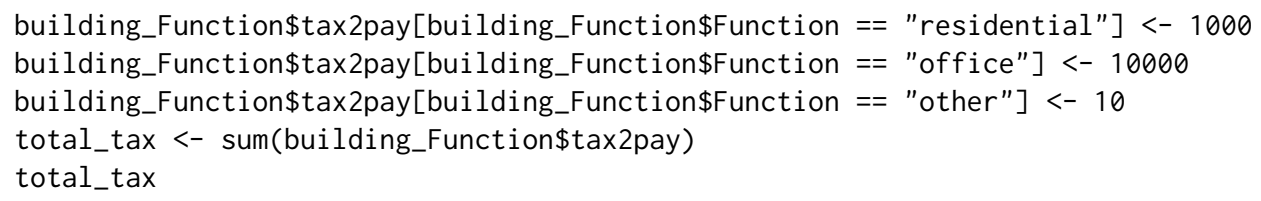

As in the previous $\mathrm{C} / \mathrm{N}$ ratio example, in order to run the propagation function, the sample of an uncertain input variable must be saved in a list. We must either coerce the existing woon_sample object or get it automatically by setting up asList = TRUE argument in genSample().

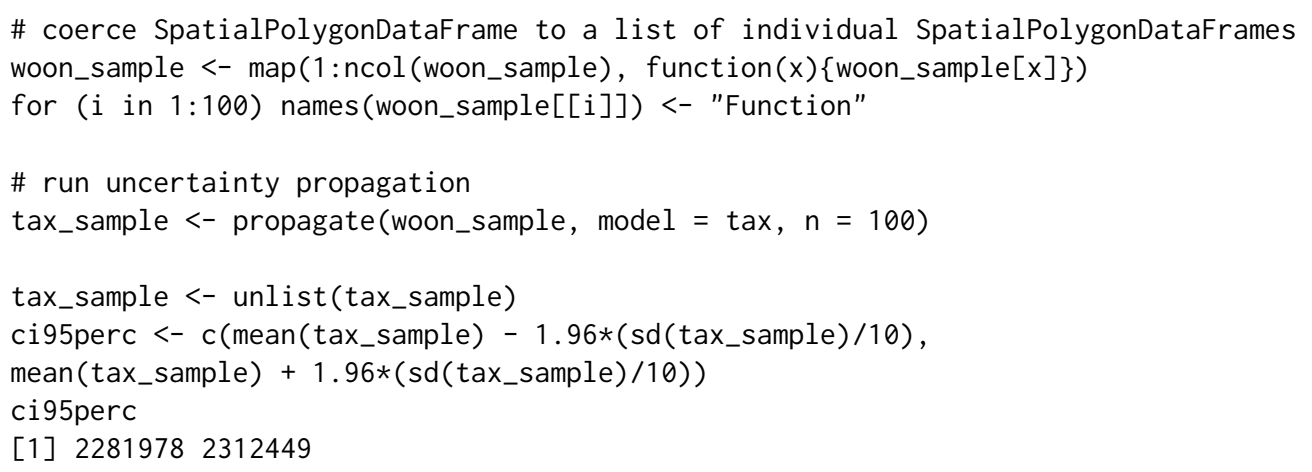

The result of the uncertainty propagation shows that on average, the city council should obtain a tax revenue of approximately $€ 2,300,000$ and that the associated $95 \%$ confidence interval is $(€ 2,281,978$, $€ 2,312,449)$.

\section{Example 3 - Uncertainty propagation analysis with a model written in C}

Environmental models are often developed in languages other than R, such as C or FORTRAN, that can significantly speed up processing. In this example, we demonstrate how to perform uncertainty analysis with a basic model written in $\mathrm{C}$.

We begin by showcasing functions to manipulate model inputs stored in ASCII files. For external models, this additional code is needed to (i) modify ASCII input files, and (ii) run the external model. For rendering ASCII input files, the mustache templating framework is available on GitHub at https://mustache.github. io, and in the R package whisker.

Suppose we have a simple linear regression model: $Y=b 0+b 1 * X$ that requires an input file input. txt. The file contains values for the two parameters $b 0$ and $b 1$ as follows:

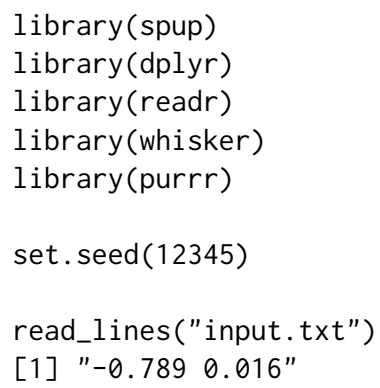

Function template() allow user to define a "container" class to store templates with model inputs. The aim of this class is to organise model input files and perform necessary checks. A print() method is available for the class "template". A template is simply an ASCII file with:

1. The additional extension .template.

2. Input that needs to be modified is replaced by mustache-style tags.

The corresponding template file should be named input.txt. template and should replace the original numbers with b0 and b1 placed in moustaches: $\{\{\ldots\}\}$.

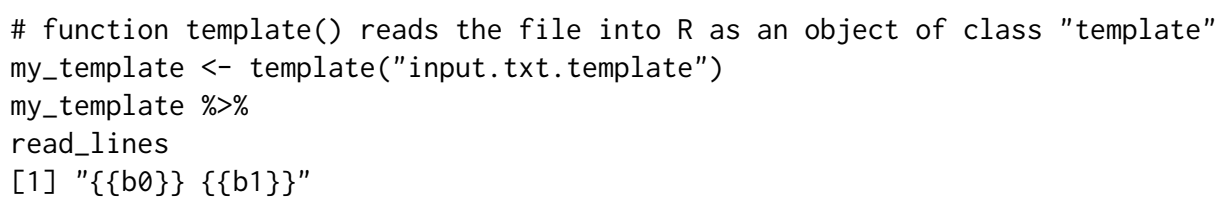


Rendering is the process of replacing the tags in moustaches with text. Rendering creates a new file, called input. txt.

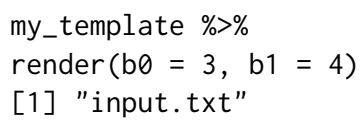

Below is an example external model written in the $C$ language. This code can be saved in a file with the .c extension, for example, 'dummy_model.c'. The model below calculates a value of a simple linear regression:

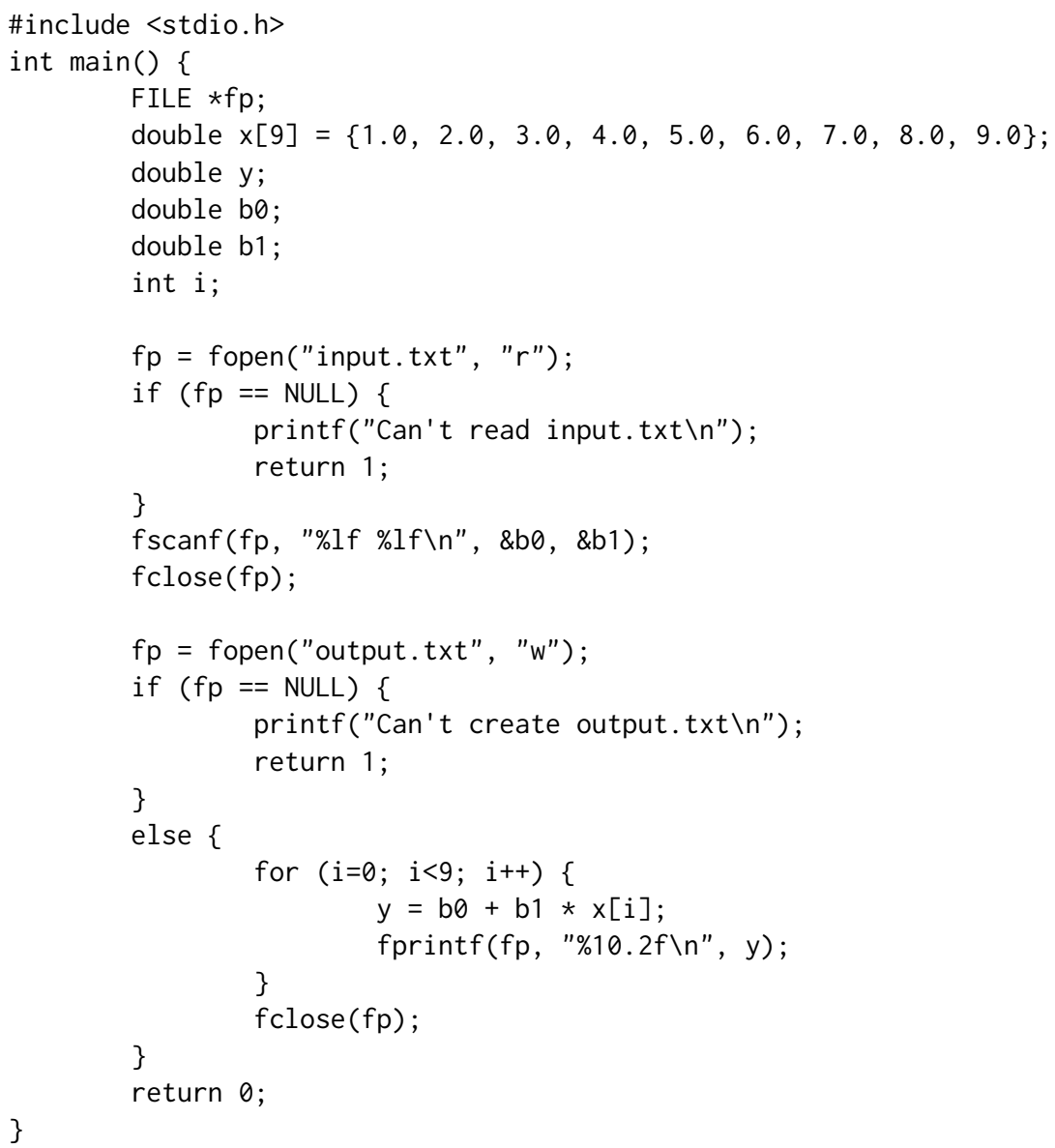

To compile this code to a MS-Windows executable one can use GCC at the DOS command prompt as follows: gcc dummy_model.c -o dummy_model. This creates a file dummy_model.exe, which can be wrapped in $\mathrm{R}$ as follows:

dummy_model <- executable("dummy_model.exe")

Running the rendering procedure passes values for $b 0$ and $b 1$ to the model, which gives:

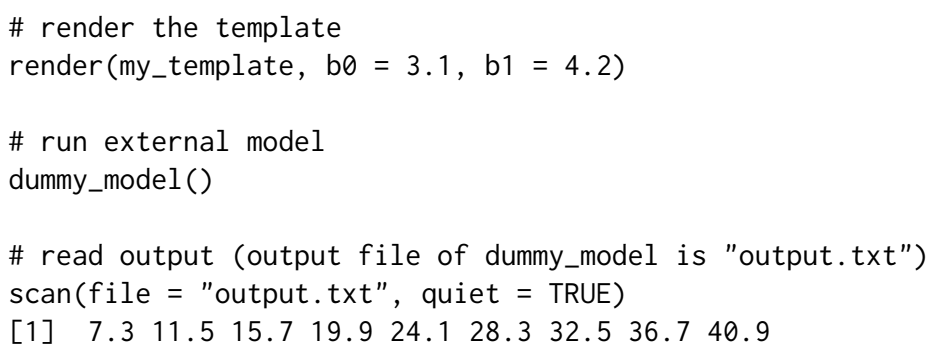

To perform the uncertainty propagation analysis, we derive multiple realizations of the model output in steps as follows: (i) render the template, (ii) run the model, (iii) read the results, and (iv) process the results. For example:

\# number of Monte Carlo runs

n_realizations $<-100$ 


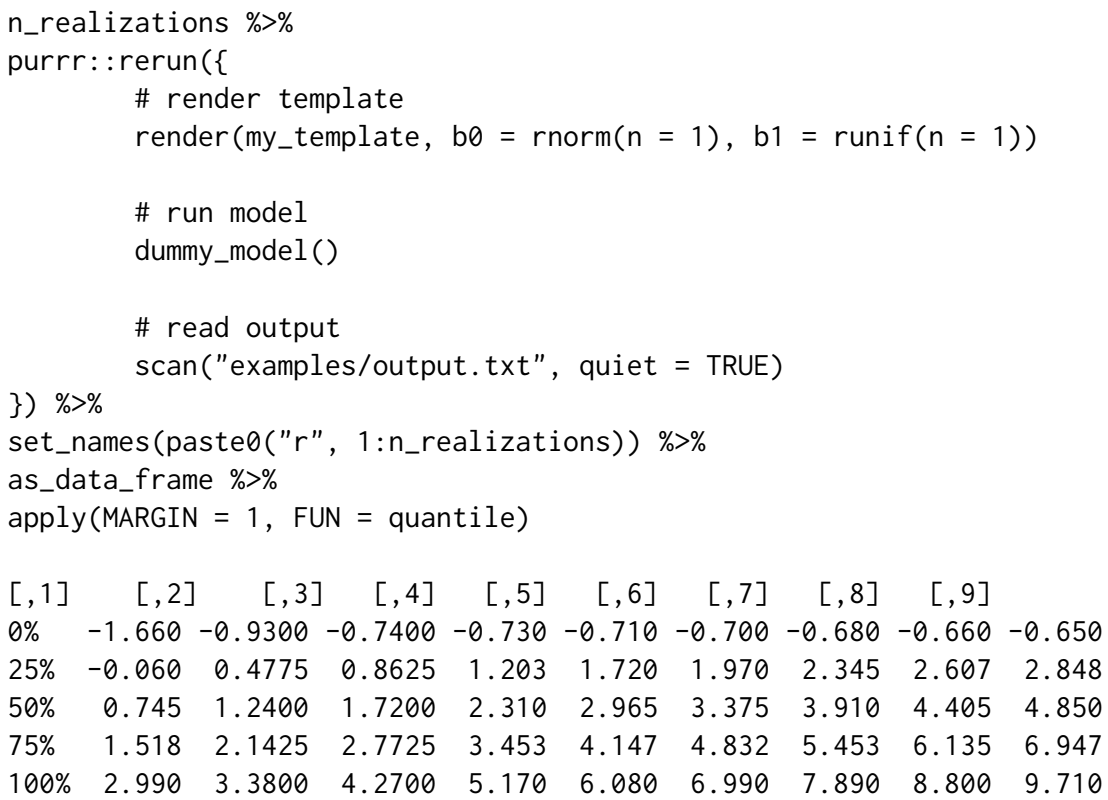

\section{Summary and future directions}

In this paper, we presented the R package spup which implements methodologies for spatial uncertainty propagation analysis. The package architecture and its core components were described and three examples were presented. We explained the package functions for examining the uncertainty propagation, starting from input data and model parameters via the environmental model to model predictions. Sources of uncertainty include model specification, stochastic simulation and propagation of uncertainty using MC techniques. We include recommendations for visualizing results. We also explained how numerical and categorical data types are handled, and how we accomodate spatial auto-correlation within an attribute and cross-correlation between attributes. The MC realizations may be used as an input to environmental models written in $\mathrm{R}$ or other programming languages that can be called from $R$. The presented examples and the vignettes included in the spup package provide insight into the selection of appropriate static uncertainty visualizations that are understandable to audiences of differing levels of statistical literacy. As an effective and easy tool, spup has potential to be used for multi-disciplinary research, model-based decision support and educational purposes.

The package could benefit from further development including facilitating uncertainty analysis with time series, one of the most common types of data in environmental studies. For uncertainty propagation, the package has implemented the MC approach with efficient sampling algorithms such as stratified random sampling; implementing Latin Hypercube sampling would complement this approach. Further direction may include interactive visualization methods could be developed via the shiny package (Chang et al., 2017). As it is based on MC methodology, spup is suitable to be used with parallel computing.

\section{Acknowledgements}

We thank Sytze de Bruin, Damiano Luzzi and Stefan van Dam for valuable contributions to the development of spup. We thank Filip Biljecki and Branislav Bajat for providing the data used in the Rotterdam and Zlatibor examples. This project has received funding from the European Union's Seventh Framework Programme for Research, Technological Development and Demonstration under grant agreement no 607000 .

\section{Authors contributions}

Kasia Sawicka is the main developer of spup and author of the text in this article. Gerard Heuvelink is a co-author of spup, he contributed to this article with his statistical and programming knowledge and edited the text. Dennis Walvoort is a co-author of spup and contributed to the development of the article. 


\section{Bibliography}

B. M. Adams, L. E. Bauman, W. J. Bohnhoff, K. R. Dalbey, M. S. Ebeida, J. P. Eddy, M. S. Eldred, P. D. Hough, K. T. Hu, J. D. Jakeman, L. P. Swiler, and D. M. Vigil. Dakota, a multilevel parallel objectoriented framework for design optimization, parameter estimation, uncertainty quantification, and sensitivity analysis: Version 5.4 user's manual. Report Sandia Technical Report SAND2010-2183, Sandia National Laboratories, 2009. [p180]

G. Andrianov, S. Burriel, S. Cambier, A. Dutfoy, I. Dutka-Malen, E. de Rocquigny, B. Sudret, P. Benjamin, R. Lebrun, F. Mangeant, and M. Pendola. Openturns, an open source initiative to treat uncertainties, risks'n statistics in 520 a structured industrial approach. In ESREL'2007 Safety and Reliability Conference, 2007. [p180]

L. Bastin, D. Cornford, R. Jones, G. B. M. Heuvelink, E. Pebesma, C. Stasch, S. Nativi, P. Mazzetti, and $\mathrm{M}$. Williams. Managing uncertainty in integrated environmental modelling: The uncertweb framework. Environmental Modelling $\mathcal{E}$ Software, 39:116-134, 2013. ISSN 1364-8152. URL https: //doi.org/10.1016/j.envsoft.2012.02.008. [p180]

D. P. Bertsekas and J. N. Tsitsiklis. Introduction to Probability. Athena Scientific, 2nd edition, 2008. ISBN 9781886529236. [p181]

A. Blake, P. Kohli, and C. Rother. Markov Random Fields for Vision and Image Processing. MIT Press, 2011. [p183]

E. W. Boyer, R. B. Alexander, W. J. Parton, C. Li, K. Butterbach-Bahl, S. D. Donner, R. W. Skaggs, and S. J. D. Grosso. Modeling denitrification in terrestrial and aquatic ecosystems at regional scales. Ecological Applications, 16(6):2123-2142, 2006. ISSN 1939-5582. URL https://doi.org/10.1890/ 1051-0761(2006)016[2123:mditaa]2.0.co;2. [p180]

J. D. Brown and G. B. M. Heuvelink. The data uncertainty engine (due): A software tool for assessing and simulating uncertain environmental variables. Computers E Geosciences, 33(2):172-190, 2007. ISSN 0098-3004. URL https://doi.org/10.1016/j.cageo.2006.06.015. [p180,182]

W. Chang, J. Cheng, J. Allaire, Y. Xie, and J. McPherson. Shiny: Web Application Framework for R, 2017. URL https://CRAN. R-project.org/package=shiny. R package version 1.0.5. [p195]

J. J. de Gruijter, D. J. Brus, M. F. P. Bierkens, and M. Knotters. Sampling for Natural Resource Monitoring. Springer, 2006. URL https://doi .org/10.1007/3-540-33161-1. [p182]

P. J. Diggle and P. J. Ribeiro. Model-Based Geostatistics. Springer-Verlag, 2007. ISBN 0387485368. URL https://doi.org/10.1007/978-0-387-48536-2. [p181,183]

D. Eddelbuettel. Cran task view: High-performance and parallel computing with r, 2017. [p182]

S. L. R. Ellison. Support for Metrological Applications, 2017. URL https://CRAN.R-project.org/ package=metRology. R package version 0.9-26-2. [p180]

R. Fisher, N. McDowell, D. Purves, P. Moorcroft, S. Sitch, P. Cox, C. Huntingford, P. Meir, and F. Ian Woodward. Assessing uncertainties in a second-generation dynamic vegetation model caused by ecological scale limitations. New Phytologist, 187(3):666-681, 2010. ISSN 1469-8137. URL https://doi.org/10.1111/j.1469-8137.2010.03340.x. [p180]

A. Genz and F. Bretz. Computation of Multivariate Normal and t Probabilities. Lecture Notes in Statistics. Springer-Verlag, Heidelberg, 2009. ISBN 9783642016882. [p182]

A. Genz, F. Bretz, T. Miwa, X. Mi, F. Leisch, F. Scheipl, and T. Hothorn. motnorm: Multivariate Normal and $t$ Distributions, 2017. URL https://CRAN. R-project.org/package=mvtnorm. R package version 1.0-6. [p182]

P. Goovaerts. Geostatistics for Natural Resources Evaluation. Oxford University Press, New York, 1997. ISBN 9780195115383. [p182, 183]

B. Gräler, E. Pebesma, and G. Heuvelink. Spatio-temporal interpolation using gstat. The R Journal, 8: 204-218, 2016. ISSN 2073-4857. [p181]

P. Hamel and A. J. Guswa. Uncertainty analysis of a spatially explicit annual water-balance model: Case study of the cape fear basin, north carolina. Hydrol. Earth Syst. Sci., 19(2):839-853, 2015. ISSN 1607-7938. URL https://doi.org/10.5194/hess-19-839-2015. [p180] 
J. M. Hammersley and D. C. Handscomb. Monte Carlo Methods. Chapman and Hall, London, 1979. [p181]

T. Hengl, D. J. J. Walvoort, A. Brown, and D. G. Rossiter. A double continuous approach to visualization and analysis of categorical maps. International Journal of Geographical Information Science, 18(2):183202, 2004. ISSN 1365-8816. URL https://doi.org/10.1080/13658810310001620924. [p184]

T. Hengl, B. Bajat, D. Blagojevic, and H. I. Reuter. Geostatistical modeling of topography using auxiliary maps. Computers $\mathcal{E}$ Geosciences, 34(12):1886-1899, 2008. ISSN 0098-3004. URL https: //doi.org/10.1016/j.cageo.2008.01.005. [p184]

T. Hengl, G. B. M. Heuvelink, and E. E. van Loon. On the uncertainty of stream networks derived from elevation data: The error propagation approach. Hydrol. Earth Syst. Sci., 14(7):1153-1165, 2010. ISSN 1607-7938. URL https://doi .org/10.5194/hess-14-1153-2010. [p180]

T. Hengl, J. Mendes de Jesus, G. B. M. Heuvelink, M. Ruiperez Gonzalez, M. Kilibarda, A. Blagotić, W. Shangguan, M. N. Wright, X. Geng, B. Bauer-Marschallinger, M. A. Guevara, R. Vargas, R. A. MacMillan, N. H. Batjes, J. G. B. Leenaars, E. Ribeiro, I. Wheeler, S. Mantel, and B. Kempen. Soilgrids250m: Global gridded soil information based on machine learning. PLOS ONE, 12(2): e0169748, 2017. URL https://doi .org/10.1371/journal . pone.0169748. [p184, 185]

G. B. M. Heuvelink. Error Propagation in Environmental Modelling with GIS. Taylor and Francis, London, 1998. [p182]

G. B. M. Heuvelink. Uncertainty in Remote Sensing and GIS, book section Analysing Uncertainty Propagation in GIS: Why is it not that Simple?, pages 155-165. John Wiley \& Sons, 2006. ISBN 9780470035269. URL https://doi.org/10.1002/0470035269.ch10. [p182]

G. B. M. Heuvelink. Error-aware gis at work: Real-world applications of the data uncertainty engine. In The International Archives of the Photogrammetry, Remote Sensing and Spatial Information Sciences, 2007. [p181, 182]

G. B. M. Heuvelink, P. A. Burrough, and A. Stein. Propagation of errors in spatial modelling with gis. International Journal of Geographical Information Systems, 3(4):303-322, 1989. ISSN 0269-3798. URL https://doi.org/10.1080/02693798908941518. [p181]

G. B. M. Heuvelink, J. D. Brown, and E. E. van Loon. A probabilistic framework for representing and simulating uncertain environmental variables. International Journal of Geographical Information Science, 21(5):497-513, 2007. ISSN 1365-8816. URL https: // doi .org/10. 1080/13658810601063951. [p181]

G. C. G. B. Hugelius. Spatial upscaling using thematic maps: An analysis of uncertainties in permafrost soil carbon estimates. Global Biogeochemical Cycles, 26(2):n/a-n/a, 2012. ISSN 1944-9224. URL https://doi.org/10.1029/2011gb004154. [p180]

R. L. Iman and W. J. Conover. A distribution-free approach to inducing rank correlation among input variables. Communications in Statistics - Simulation and Computation, 11(3):311-334, 1982. ISSN 0361-0918. URL https://doi.org/10.1080/03610918208812265. [p182]

H. I. Jager, A. W. King, N. H. Schumaker, T. L. Ashwood, and B. L. Jackson. Spatial uncertainty analysis of population models. Ecological Modelling, 185(1):13-27, 2005. ISSN 0304-3800. URL https://doi.org/10.1016/j.ecolmode1.2004.10.016. [p180]

C. Kinkeldey, A. M. MacEachren, and J. Schiewe. How to assess visual communication of uncertainty? a systematic review of geospatial uncertainty visualisation user studies. The Cartographic Journal, 51(4):372-386, 2014. ISSN 0008-7041. URL https://doi .org/10.1179/1743277414y. 0000000099. [p184]

A. M. Lechner, C. M. Raymond, V. M. Adams, M. Polyakov, A. Gordon, J. R. Rhodes, M. Mills, A. Stein, C. D. Ives, and E. C. Lefroy. Characterizing spatial uncertainty when integrating social data in conservation planning. Conserv Biol, 28(6):1497-511, 2014. ISSN 0888-8892. URL https: //doi.org/10.1111/cobi.12409. [p180]

P. A. W. Lewis and E. J. Orav. Simulation Methodology for Statisticians, Operations Analysts, and Engineers, volume 1. Wadsworth \& Brooks/Cole, 1989. ISBN 9780534094508. [p181]

E. Leyva-Suarez, G. S. Herrera, and L. M. de la Cruz. A parallel computing strategy for monte carlo simulation using groundwater models. Geofísica Internacional, 54(3):245-254, 2015. ISSN 0016-7169. URL https://doi.org/10.1016/j.gi.2015.04.020. [p182] 
A. M. MacEachren. Visualizing uncertain information. Cartographic Perspective, 13:10-19, 1992. [p184]

A. M. MacEachren, A. Robinson, S. Hopper, S. Gardner, R. Murray, M. Gahegan, and E. Hetzler. Visualizing geospatial information uncertainty: What we know and what we need to know. Cartography and Geographic Information Science, 32(3):139-160, 2005. ISSN 1523-0406. URL https://doi.org/10.1559/1523040054738936. [p184]

S. Marelli and B. Sudret. Vulnerability, Uncertainty, and Risk, book section UQLab: A Framework for Uncertainty Quantification in Matlab, pages 2554-2563. American Society of Civil Engineers, 2014. ISBN 978-0-7844-1360-9. URL https://doi .org/10.1061/9780784413609.257. [p180]

M. Muthusamy, A. Schellart, S. Tait, and G. B. M. Heuvelink. Geostatistical upscaling of rain gauge data to support uncertainty analysis of lumped urban hydrological models. Hydrol. Earth Syst. Sci. Discuss., 2016:1-27, 2016. ISSN 1812-2116. URL https://doi .org/10. 5194/hess-2016-279. [p180]

C. O. Nijhof, M. A. Huijbregts, L. Golsteijn, and R. van Zelm. Spatial variability versus parameter uncertainty in freshwater fate and exposure factors of chemicals. Chemosphere, 149:101-7, 2016. ISSN 0045-6535. URL https://doi.org/10.1016/j.chemosphere.2016.01.079. [p180]

L. Nol, G. B. M. Heuvelink, A. Veldkamp, W. de Vries, and J. Kros. Uncertainty propagation analysis of an n2o emission model at the plot and landscape scale. Geoderma, 159(1-2):9-23, 2010. ISSN 0016-7061. URL https://doi.org/10.1016/j.geoderma.2010.06.009. [p180]

E. J. Pebesma. Multivariable geostatistics in s: The gstat package. Computers E Geosciences, 30(7): 683-691, 2004. ISSN 0098-3004. URL https://doi .org/10.1016/j.cageo. 2004.03.012. [p181, 182]

E. J. Pebesma and G. B. M. Heuvelink. Latin hypercube sampling of gaussian random fields. Technometrics, 41(4):303-312, 1999. ISSN 00401706. URL https:// doi .org/10.2307/1271347. [p182]

D. Pennock, T. Yates, A. Bedard-Haughn, K. Phipps, R. Farrell, and R. McDougal. Landscape controls on $\mathrm{n} 2 \mathrm{o}$ and ch 4 emissions from freshwater mineral soil wetlands of the canadian prairie pothole region. Geoderma, 155(3-4):308-319, 2010. ISSN 0016-7061. URL https://doi.org/10.1016/j . geoderma.2009.12.015. [p180]

F. Pianosi, F. Sarrazin, and T. Wagener. A matlab toolbox for global sensitivity analysis. Environmental Modelling E Software, 70:80-85, 2015. ISSN 1364-8152. URL https://doi .org/10.1016/j . envsoft. 2015.04.009. [p180]

R. E. Plant. Spatial Data Analysis in Ecology and Agriculture Using R. CRC Press, 2012. ISBN 1439819130. [p181]

A. Saltelli, S. Tarantola, and F. Campolongo. Sensitivity anaysis as an ingredient of modeling. Statistical Science, pages 377-395, 2000. ISSN 0883-4237. URL https://doi.org/10.1214/ss/1009213004. [p182]

K. Sawicka, G. B. M. Heuvelink, and D. J. J. Walvoort. Spup: Uncertainty Propagation Analysis, 2017. R package version 0.1-1. [p180, 185]

G. I. Schueller and H. J. Pradlwarter. Computational stochastic structural analysis (cossan) - a software tool. Structural Safety, 28(1-2):68-82, 2006. ISSN 0167-4730. URL https://doi .org/10. $1016 / j$.strusafe.2005.03.005. [p180]

A.-N. Spiess. Propagate: Propagation of Uncertainty, 2014. URL https://CRAN. R-project. org/package= propagate. R package version 1.0-4. [p180]

I. Ucar. Errors: Error Propagation for R Vectors, 2017. URL https://CRAN.R-project. org/package= errors. R package version 0.0.2. [p180]

M. Van Oijen, J. Rougier, and R. Smith. Bayesian calibration of process-based forest models: Bridging the gap between models and data. Tree Physiology, 25(7):915-927, 2005. ISSN 0829-318X. URL https://doi.org/10.1093/treephys/25.7.915. [p181]

E. I. Vanguelova, E. Bonifacio, B. De Vos, M. R. Hoosbeek, T. W. Berger, L. Vesterdal, K. Armolaitis, L. Celi, L. Dinca, O. J. Kjønaas, P. Pavlenda, J. Pumpanen, U. Püttsepp, B. Reidy, P. Simončič, B. Tobin, and M. Zhiyanski. Sources of errors and uncertainties in the assessment of forest soil carbon stocks at different scales-review and recommendations. Environmental Monitoring and Assessment, 188(11): 630, 2016. ISSN 1573-2959. URL https://doi.org/10.1007/s10661-016-5608-5. [p180]

H. Wackernagel. Multivariate Geostatistics: An Introduction with Applications. Springer, 2003. [p186] 
R. Webster and M. A. Oliver. Geostatistics for Environmental Scientists. John Wiley \& Sons, 2nd edition, 2007. [p181, 187]

X. Yu, A. Lamacova, C. Duffy, P. Kram, and J. Hruska. Hydrological model uncertainty due to spatial evapotranspiration estimation methods. Computers $\mathcal{E}$ Geosciences, 90, Part B:90-101, 2016. ISSN 0098-3004. URL https://doi.org/10.1016/j.cageo.2015.05.006. [p180]

\author{
Kasia Sawicka \\ Centre for Ecology \& Hydrology \\ Deiniol Road \\ Bangor \\ LL57 2UW \\ United Kingdom \\ (Previously at: Soil Geography and Landscape Group \\ Wageningen University \\ PO Box 47 \\ 6700AA Wageningen \\ The Netherlands) \\ ORCiD: 0000-0002-7553-3149 \\ katwic55@ceh.ac.uk \\ Gerard B.M. Heuvelink \\ Soil Geography and Landscape group \\ Wageningen University \\ PO Box 47 \\ 6700AA Wageningen \\ The Netherlands \\ ORCiD: 0000-0003-0959-9358 \\ gerard.heuvelink@wur.ml \\ Dennis J.J. Walvoort \\ Wageningen Environmental Research \\ PO Box 47 \\ 6700AA Wageningen \\ The Netherlands \\ dennis.walvoort@wur.nl
}

